\title{
Design and Development of an Automated Monitored Hand Hygiene System to Curb Infection Spread in Institutional Settings during COVID-19 Pandemic
}

Ashish Karn, Rithvik Kanchi, Shashank Singh Deo

Department of Mechanical Engineering, School of Engineering, University of Petroleum and Energy

Studies, Energy Acres, Bidholi, Dehradun, Uttarakhand, India 248007

* Email addresses of the corresponding author: akarn@ddn.upes.ac.in

\begin{abstract}
Considering the current scenario of Coronavirus outbreak and the post-pandemic situation, the need of a robust hand hygiene program assumes utmost importance, particularly in institutional settings such as education and healthcare. As different nations all across the globe lift the lockdown restrictions and as life springs back to normalcy, organizations, in order to quell the apprehensions and concerns of its workers, may have to institute newer paradigms to curb infection and generate awareness. Prior reported research shows that although an adequately designed health hygiene system may be helpful in curbing the infection spread, a major issue stems from the lack of adherence, by a section of individuals in any institution. In such a situation, it becomes absolutely imperative to track the compliance by different individuals, so that educational interventions may be targeted for the concerned group of workers. Further, currently, no low-cost compact systems exist that can provide for the hand hygiene requirements for a group of people, which are "touchfree", automated and which are monitored. To bridge this gap, we propose the design and development of an automated monitored hand hygiene system to curb infection spread in institutional settings. It is designed in such a way that it would provide for hand sanitization of four people at a time per machine, simultaneously ensuring social distancing between them. The proposed product will not only reduce the apprehensions of the workers in an institutions by providing for solutions to curb infection spread, but will also be economical and aesthetically compact to be deployed at multiple locations within an organization, thus providing for a much safer workplace during the post-lockdown phase.
\end{abstract}

Keywords: COVID-19; Hand hygiene; sanitizer, virus, disinfectant 


\section{Introduction}

In the event of a pandemic such as the COVID-19 outbreak, it requires a multi-pronged approach to address the issues including both pharmacological and non-pharmacological interventions, apart from the massive education of the general populace and communities. While a pharmacological intervention may refer to a drug or a vaccine, non-pharmacological interventions could refer to a number of strategies such as hand hygiene or facemasks case isolation in home, social distancing, voluntary home quarantines, closure of educational institutions [1]. Of the different kinds of such non-pharmacological interventions, hygienic hand antisepsis is one of the most important intervention to prevent the transmission of pathogenic microorganisms and can reduce infection spread, even among high risk populations [2]. Evidence from the literature showed that frequent hand-washing would reduce the risk of viral transmission by $55 \%$ [3, 4]. Further, an appropriate hand washing intervention can even reduce the risk of infection by $6 \%$ to $44 \%$, by impairing the transmission cycle and thus hand hygiene education has an indispensable role in the prevention of infectious diseases. [5]

Particularly, in an institutional setting, such as an educational institutions, industry, offices, healthcare facilities etc, ensuring the correct hand hygiene promotion program is extremely important. Such a hand hygiene scheme should preferably be "touch-free", so that the chances of viral transmission are minimized. A plausible solution to ensure the hand-hygiene of individuals is using an automated hand sanitizers. However, this is not enough in an institutional setting where individuals throng in large numbers, particularly during peak hours, exacerbated by the apathy and unwillingness of individuals to comply to a standard hand-hygiene protocol. There is clearly a need for a technological intervention which not only facilitates hand sanitization, but also maintains sufficient physical distance between people, and necessitates compliance by different members of institution. Such a multimodal hand hygiene antisepsis program can also identify institution workers with poor compliance to hand hygiene regulations, and thus assist in the development of educational interventions at a targeted sub-population within an institution. [6]

During the outbreak of a pandemic, social distancing, lockdowns and work-from-home regulations have been released by a majority of the countries, in a bid to reduce the infection spread of the Coronavirus pandemic. However, as the lockdowns are lifted, there is an increased apprehension and concern among organizations to safeguard their workers against the pandemic by instituting systems which can protect individuals against the viral transmission. Under such a situation, the "touch-based" hand sanitizers are susceptible to cause human-to-human transmission, and even small scale automated sanitizers are not enough to bridge the gap, without a large scale hand antisepsis system. Further, these large scale systems, should ensure enough physical distance between different users, should monitor the usage, and should be cheap enough to be installed at multiple locations within an organization. Although there are some manufacturers of automatic hand hygiene monitoring systems in USA such as Biovigil, DebMed, Gojo Industries etc., there are no such existing industry in India. These systems typically employ some sort of wireless communications technology such as a WiFi, RFID badges, both of these or even none. Many of these systems require the subject to wear a badge/tag or to wear it on the wrist. However, there are no currently existing systems which are low-cost and that institutions can deploy without having the workers to wear a badge/tag/ wrist bands etc. and simultaneously provide monitoring. Thus, there is clearly a need for automated monitored hand-hygiene antisepsis systems which are easily customizable and so cheap that many of these units could be deployed within a single institution. Here, we propose the design and development of such a system which is extremely simple to build. 


\section{Considerations in the design of Hand hygiene systems for community settings during COVID-19 pandemic}

There are various important considerations for the design of hand hygiene systems for community and health care systems, which can be outlined as under:

\subsection{Automation}

Coronaviruses are enveloped viruses and in particular SARS-CoV-2, the virus that causes COVID19 is believed to spread mainly through human-human transmission via respiratory droplets or through other routes such as contact with contaminated fomites and inhalation of aerosols, produced during aerosol generating procedures.[2] A recent review during Coronavirus pandemic has shown that corona viruses survive on surfaces for a period ranging from 2 hours to 9 days. [7] The conventional hand sanitizers are touch-based and its use in institutional settings may pose a significant health hazard by promoting infection spread.

\subsection{Compliance and Monitoring}

Although the hand-hygiene has been acknowledged as the most important component of an adequate infection control scheme, the major bottleneck to its success comes from the paucity of adherence to the proper protocols. The poor compliance has often been attributed to such factors such as lack of time, hand dryness, hand irritation, sceptical attitude of people regarding its usefulness, lack of monitoring, perceptions regarding low risk of cross-infection, inconvenience, and the misconceived belief that protection is offered by gloves alone [8]. To increase the level of adherence to hand hygiene regimen, the hand hygiene compliance should be monitored and the prior literature suggests that a monitored protocol yields much higher compliance. [6]

\subsection{The need to ensure 'Social distancing'}

The implementation of a hand hygiene program with a single sanitizer unit, whether "touch-based" or automated is fraught with risk, since it does not promote physical distancing between individuals. Any possible design solution must ensure that different "automated" hand hygiene units are separated and institution workers can only approach through a queue. Further, such systems must be compact, and so can be placed at a suitable entry location in the institutions such as reception, main entry points etc.

\subsection{Format}

There are various formats of hand hygiene strategies such as a medicated soap solution, antiseptic agent, detergents, waterless antiseptic agents, alcohol-based hand rub (ABHRs) or it could be a generic preparation containing one or more types of alcohols, humectants, excipients etc. in the form of a gel, foam or liquid [9]. Gels and foams provide a less clean-feeling and slower to dry at higher doses, whereas liquids yield a smoother, cleaner, a greater moisturized feel, although there is a greater difficulty in handling these liquids which outweighs these benefits. Thus, the key desirable properties are fast absorption, moisturized and clean hand feel, lack of stickiness, and low odour and hence gels and foams are more preferred over liquids. [10]

Using ABHR over handwashing with soap has several key advantages including better effectiveness, faster action, convenience in use and also lesser and milder reactions by the skin compared to soap and water. Contrary to the antimicrobials used in soaps, bacterial or viral resistance to alcohol at the typical concentrations used in ABHRs, is much smaller. Usage of 
ABHRs have another distinct advantage: it reduces the water requirements, and is advantageous in water-deficient locations. [11]

\subsection{Formulation}

There are various formulations proposed by WHO which can be used as an antiseptic or disinfectants against viruses including soap and water, ABHRs, chlorine solutions, Iodine and Iodophors, Hydrogen peroxide etc. It has been reported that the virucidal efficiency of alcohol against certain viruses, such as a Norovirus varied depending upon the contact time, formulation and concentration. [12] Typically, washing hands with soap and water works best when hands are visibly dirty and it should be done for 40-60 seconds. However, if hands are not visibly dirty, using ABHRs for 20-30 seconds is the preferred method of cleaning. WHO recommended formulations contain either $75 \% \mathrm{v} / \mathrm{v}$ isopropanol or $80 \% \mathrm{v} / \mathrm{v}$ ethanol. [9] ABHRs containing $60-90 \%$ ethyl alcohol or isopropyl solutions in water can be used for cleaning since it contains strong virucidal agents inactivating all the lipophilic viruses. A recent review by Kampf (2020) reported that 62$71 \%$ ethanol solution can efficiently inactivate human coronaviruses within 1 minute. WHO typically recommends $70 \%$ ethyl alcohol to disinfect small areas.

Chlorine solutions such as $0.05 \%$ chlorinated water can be used as a suitable disinfectant for hand washing. Kampf et al. (2020) reported that $0.1 \%$ sodium hypochlorite solutions can efficiently inactivate human coronaviruses within 1 minute. Similarly, Chlorhexidine (0.5-4\% concentration) can also be used to decrease the infectivity of the enveloped viruses and thus are suitable for hand washing, although some studies have reported its inability to disinfect enveloped human coronavirus [13]. Commercially available formulations of Povidone Iodine are effective virucidal activities, for instance Iodophors ( $0.5-10 \%$ concentration) can be used for hand washing to reduce the infection potential of enveloped viruses. Even Hydrogen Peroxide at $0.5 \%$ concentration has been reported to decrease the infection capability of human coronaviruses, when used for a minute [14]

\subsection{Volume}

Some studies have reported that in a hospital setting, the volume of ABHR dispensed by the hospital's automated dispenser is close to $1.1 \mathrm{~mL}$ per dose and the ABHR dose is inversely related with the number of applications of ABHR per shift, but has no relationship with the hand size, although there is a concern that it might create a risk for people with larger hand size. [15]

\subsection{Time}

A study reported that hand rubbing for 15 seconds yielded results at par with hand rubbing for 30 seconds and no significant difference with respect to bacterial counts on hands were found. [16] Similarly, a comparison of ABHR and the traditional hand-washing with regard to the time required for complete disinfection revealed a value of 72 seconds and 40 seconds, respectively indicating that ABHRs are more time-effective as compared to the traditional hand-washing with soap and water. [8]

\subsection{Economics of hand hygiene}

Interestingly, a recent study conducted in the dental setting compared the cost associated with traditional hand washing against an ABHR protocol and the corresponding time required to implement acceptable hand hygiene levels. As far as the cost of hand hygiene is concerned, it was 
found out that the hand sanitation regimen with soap and water costs $\$ 0.052$, as compared to $\$ 0.023$ with ABHR. These results indicate that ABHR protocol is less expensive as compared to a traditional hand washing. [8]

\subsection{Skin reactions}

According to WHO, there are two major types of skin reactions pertaining to hand antisepsis program. The first kind could be mild to debilitating and commonly referred to as irritant contact dermatitis. It can include irritation, dryness, itching or even cracking and bleeding. The other kind of skin reaction, alluded to as the allergic contact dermatitis, is scarce and usually stems from an allergy to some ingredient in the disinfectant liquid. [17]

\section{Proposed design and its innovative features}

The proposed product 'Automated Monitored Hand-hygiene system for Institutional Settings' is intended to be used in educational institutions, corporate houses, healthcare facilities and other community settings such as shopping complexes etc.. which report a considerable amount of influx of people, particularly at some peak hours during the day. The proposed design is a compact, lowcost alternative which not only dispenses disinfecting liquids at multiple ports near the entrance gates, but also monitors the compliance by different users. In addition, the volume of the dispensed liquid, its format and formulation is strictly in accordance with the findings reported in the literature.
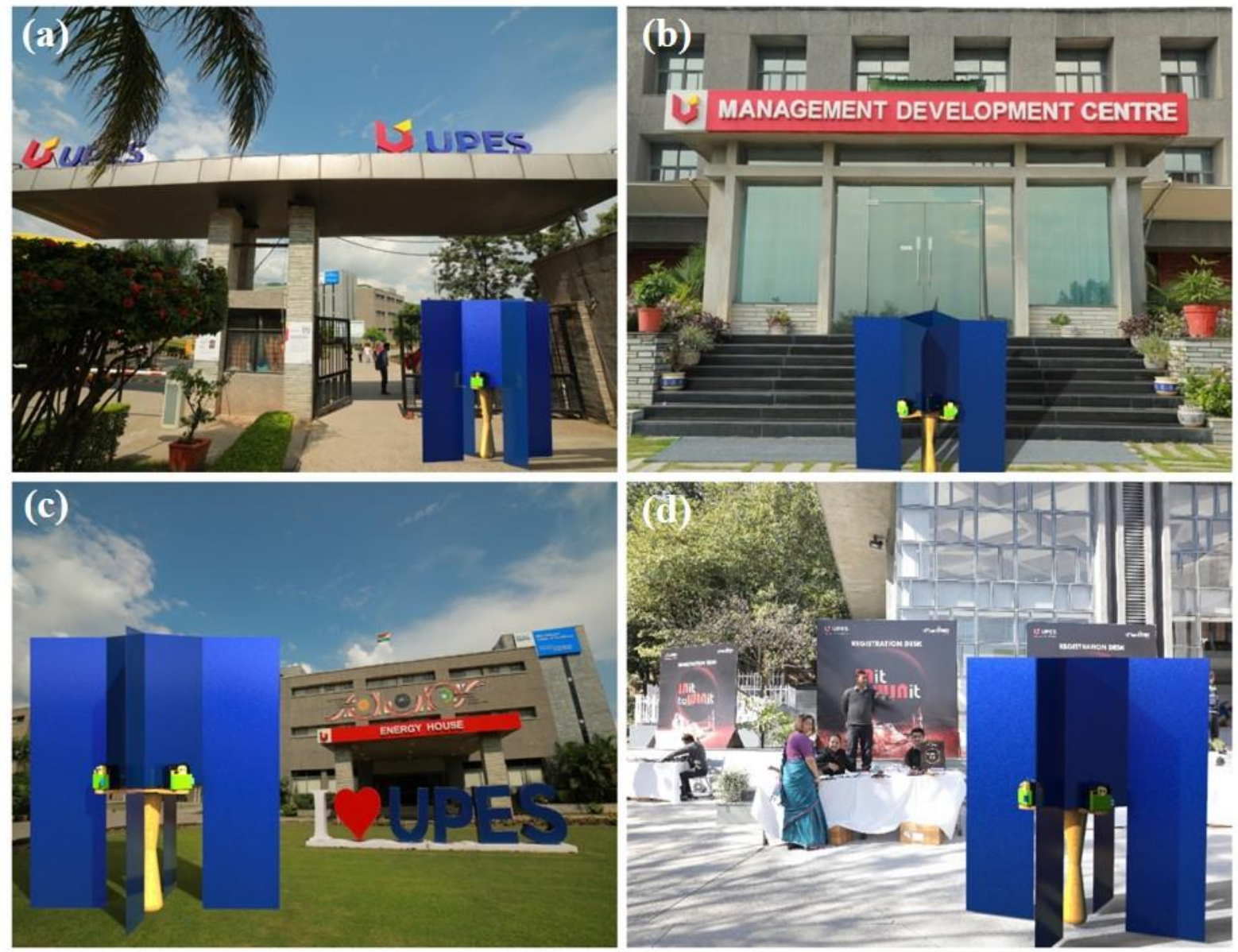

Figure 1: A schematic of the developed hand-hygiene system product deployed at four representative locations in a University campus where there is a large influx of students, such as (a) The main gate, (b) A management office, (c) In front of University reception and (d) in front of 
University gymnasium and auditorium. Note that the adjacent dispensing units have tall separators between them, extending outwards.

Figure 1 shows a schematic of the proposed design ant different gateways in a typical University campus - at the main entrance, near the University central office reception, outside the University gymnasium and out of a management office. Typically, these are the prime zones where the congregation of students, faculty/staff is expected, particularly during the peak hours. It is evident that several of these proposed design units could be installed in an organization such as a University. With its compact design, the product does not take a considerable space, and the presence of the tall separators between the adjacent dispensing units ensure that individuals don't throng together, breaking the social distancing norms. Rather, individuals have to walk in a queue from the four directions, to approach the dispensing units. Further, the four separators have been designed extending outwards, so that the users can walk in and out of line from the either side, reducing chaos and ensuring safe distance between individuals.

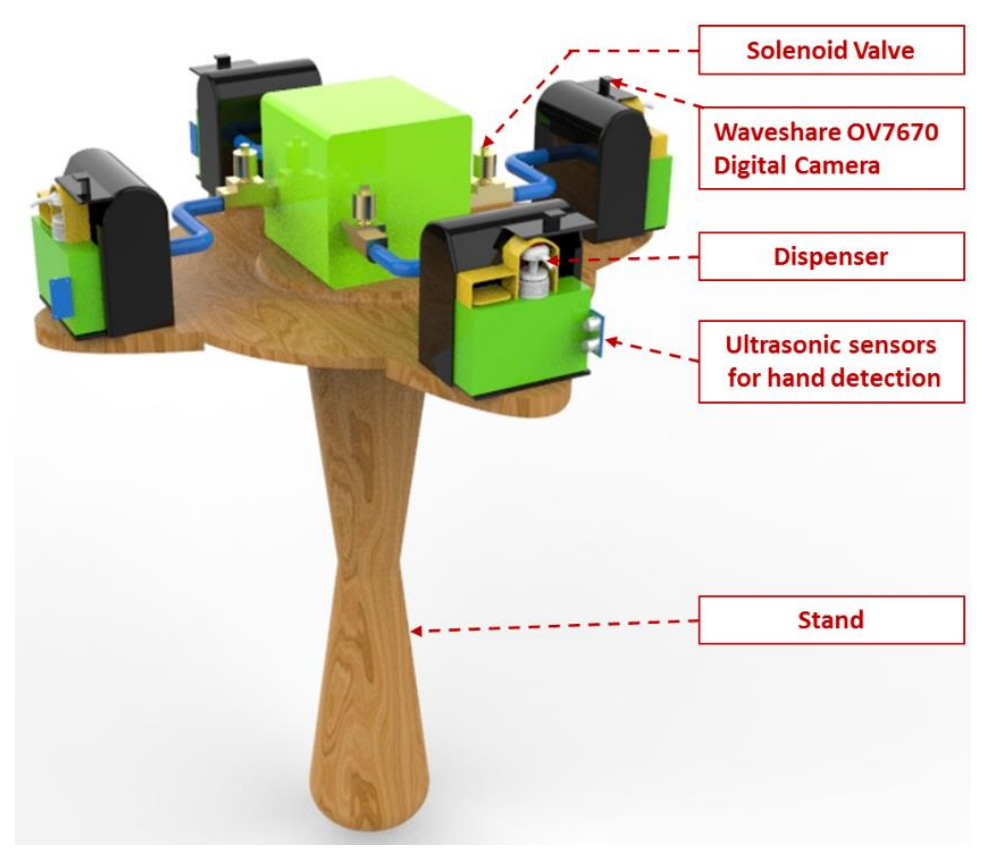

Figure 2: An isometric view of the proposed automated and monitored hand hygiene system. The main tank is shown smaller in height, for the sake of clarity.

Figure 2 shows a closer view of the designed product. For getting the disinfectant liquid, the user has to place their hand under the nozzle of the dispenser, whereupon the ultrasonic sensors detect the presence of a hand and triggers the dispensing process out of a sub-tank. Figure 3 shows a top view of the product through which it is clearly evident that the four dispensing units with their subtanks lie at the periphery of a central sanitizer tank (also referred to as 'main tank'). The main tank is a large tank and is expected to stock up the sanitizer solution for almost a week. The volume of the main tank is estimated to be 60 litres, based upon a simple calculation. Assuming a single person uses the same product at the same location thrice in a day, and based on the reported research literature, it has been determined that $1.2 \mathrm{ml}$ of solution is considered to suffice for most efficient disinfection. Thus, for an organizational strength of 3000 people per product, this consumption amounts to $10.8 \mathrm{~L}$ for every single day, or $54 \mathrm{~L}$ per work week. An excess of $6 \mathrm{~L}$ solution is ensured to account for the sanitization by the guests and visitors. The main tank can be refilled easily, simpl by replacing the top cover. 


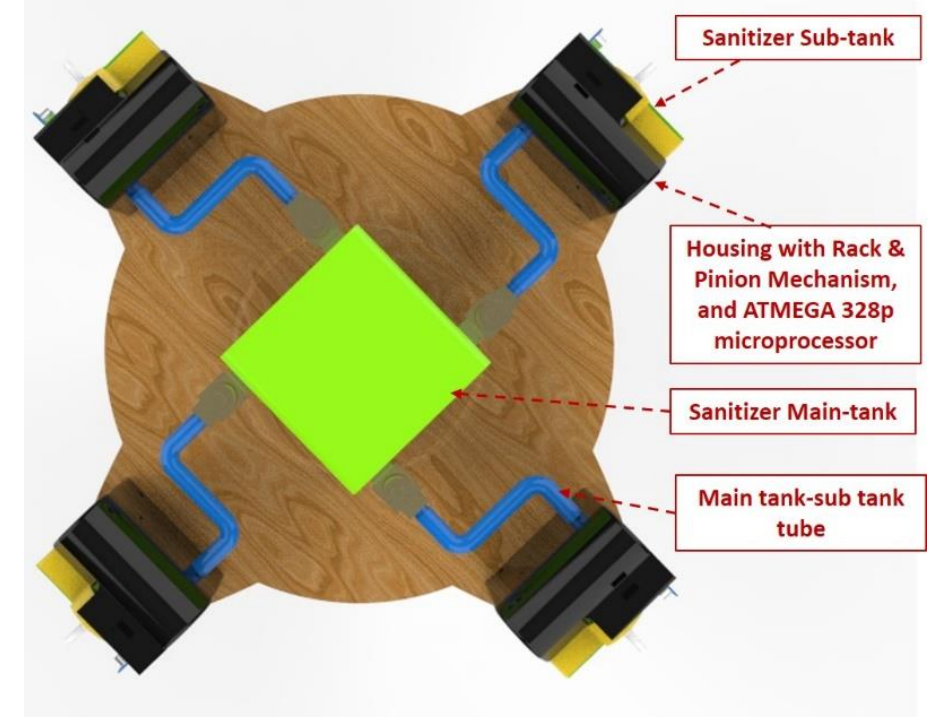

Figure 3: A top view of the proposed automated and monitored hand hygiene system

The proposed product is recommended to work with ABHRs, although it can work with any other solution. The amount of the ABHR dispensed per usage has been fixed at $1.2 \mathrm{ml}$, which has been found to be more than the required dosage for complete hand disinfection.

\section{Working}

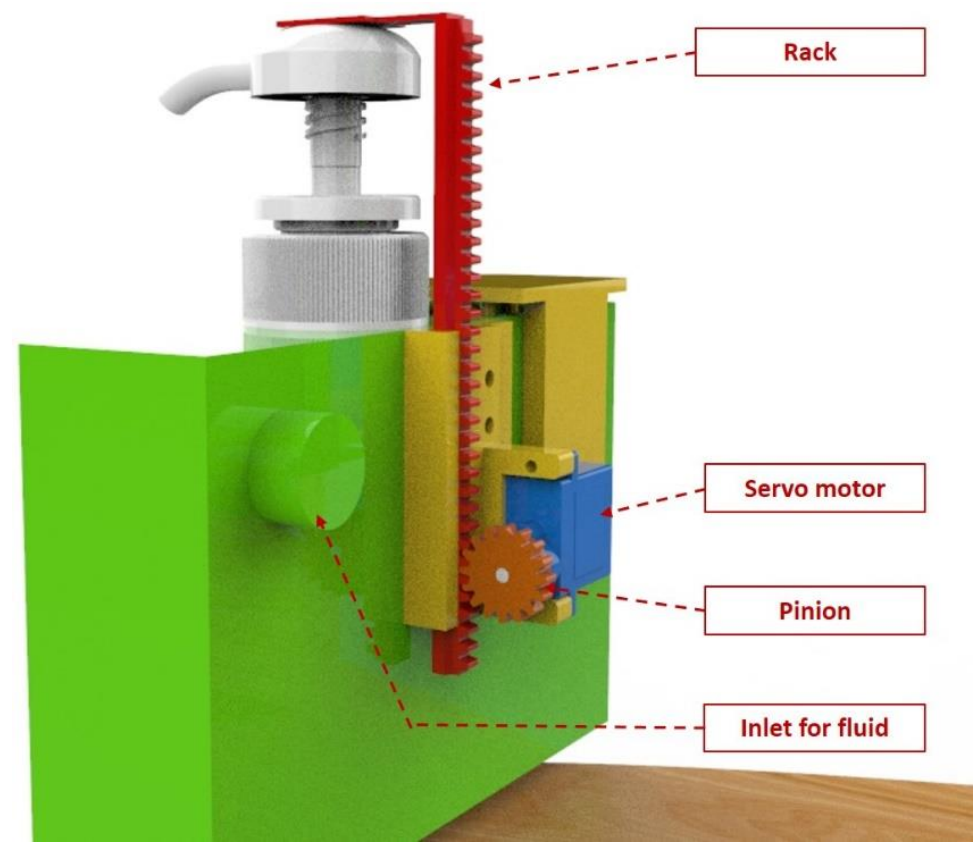

Figure 4: A backside isometric view of the dispenser showing rack and pinion mechanism.

The proposed design uses a basic control system with a feedback loop for the actuation/ automation of the device. An ultrasonic sensor senses the hand and a signal is relayed to the camera module and the servomotor simultaneously, which performs an angular rotation, clockwise or anticlockwise as shown in Figure 4. The power acquired by this motion is transferred to the single displacement pump (or the pump on the dispenser) which ejects sanitizer or hand wash via a rack 
and a pinion. The control is accomplished through an Atmega328p microprocessor which is initial programmed in Arduino IDE (Integrated development environment). Figure 5 shows that the refilling is accomplished by a generic $12 \mathrm{~V}$ solenoid valve, an ultrasonic sensor which is used to measure the sanitizer level in the sub-tank for hand sensing and pipes of favourable length to help connect the solenoid valve of the main tank and the solenoid valve of the sub tank.

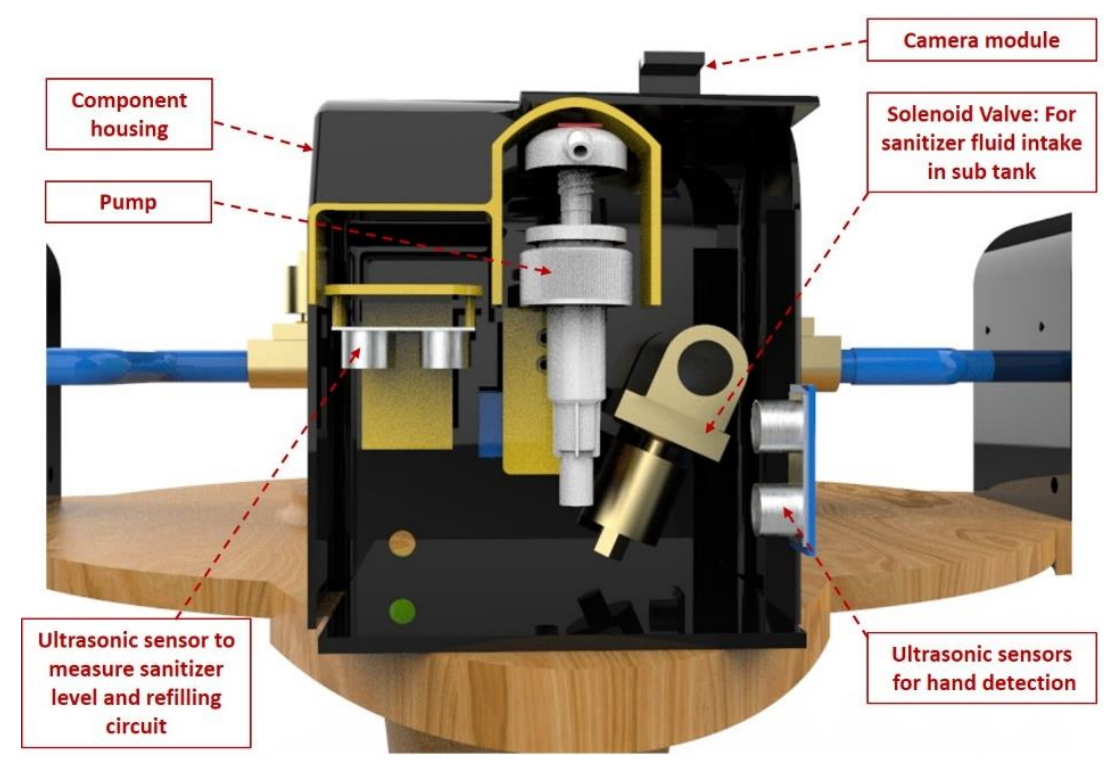

Figure 5: A cut-section view inside the housing, near the dispenser

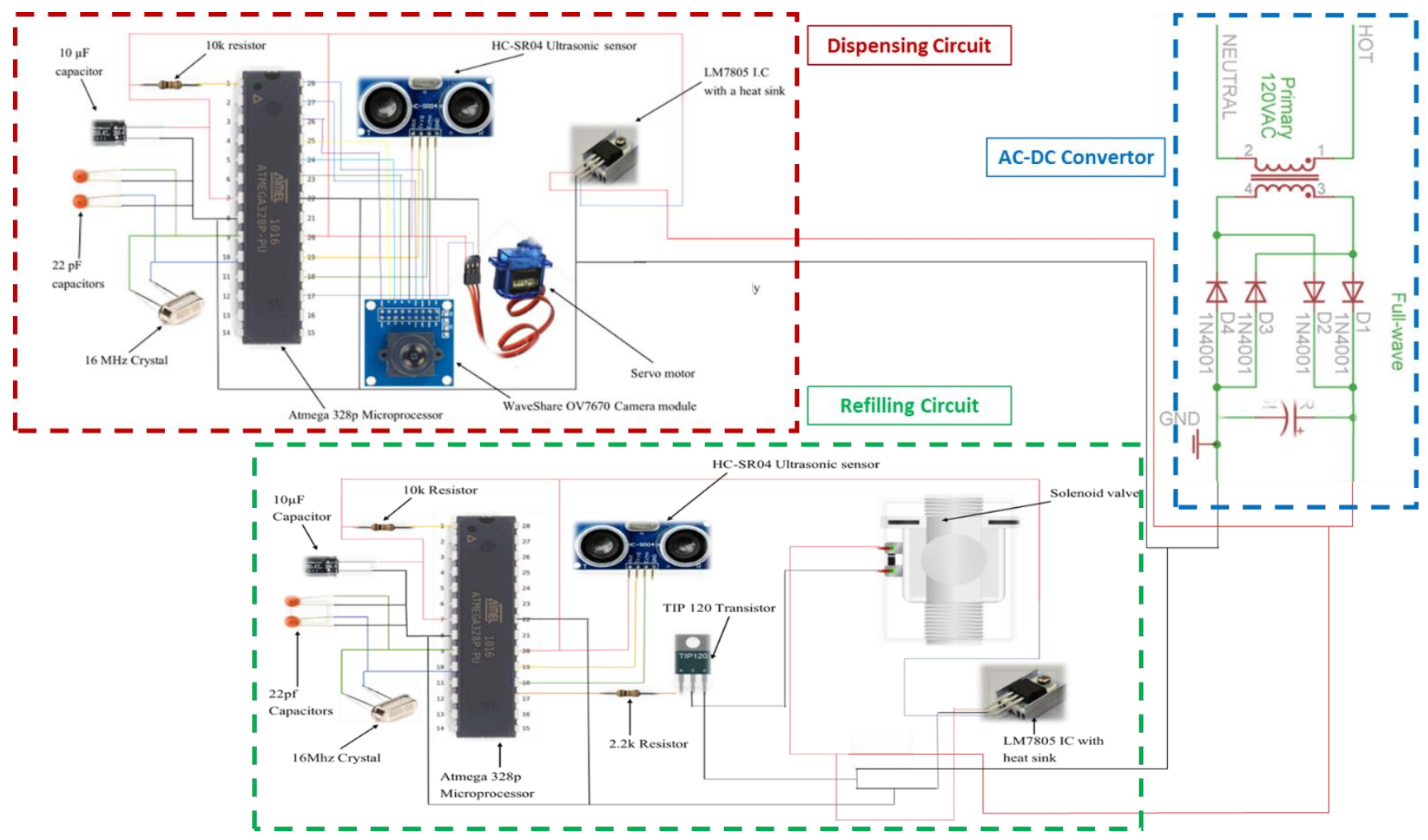

Figure 6: A comprehensive electrical circuitry of the proposed product 
Figure 6 shows a comprehensive electrical circuitry of the proposed product. It broadly consists of three sub-circuits: dispensing and monitoring circuit, refilling circuit and an AC-DC converter. Since all the required components such as the microprocessor, ultra sonic sensor and the servo motor all operate on a 5V supply, an AC-DC converter with capability to step down the voltage to $12 \mathrm{~V}$ is used. Next, figure 7 shows an electrical circuitry of the dispensing process. With the help of an LM7805 IC (which is nothing but a voltage divider circuit) with a heat sink, this voltage is further dropped to $5 \mathrm{~V}$ operating voltage. The Atmega $328 \mathrm{p}$ microprocessor is then mounted over a honeycomb board and with supporting units such as a $16 \mathrm{MHz}$ crystal to keep track of time, while some capacitors and resistors are employed to maintain the required current input for the processor to work. The ultrasonic sensor is used for the detection of the proximity of any kind and the servomotor responds to the output generated by the ultrasonic sensor. Further, with the appropriate code the values for desired motor rotation and at a desired proximity can be set.

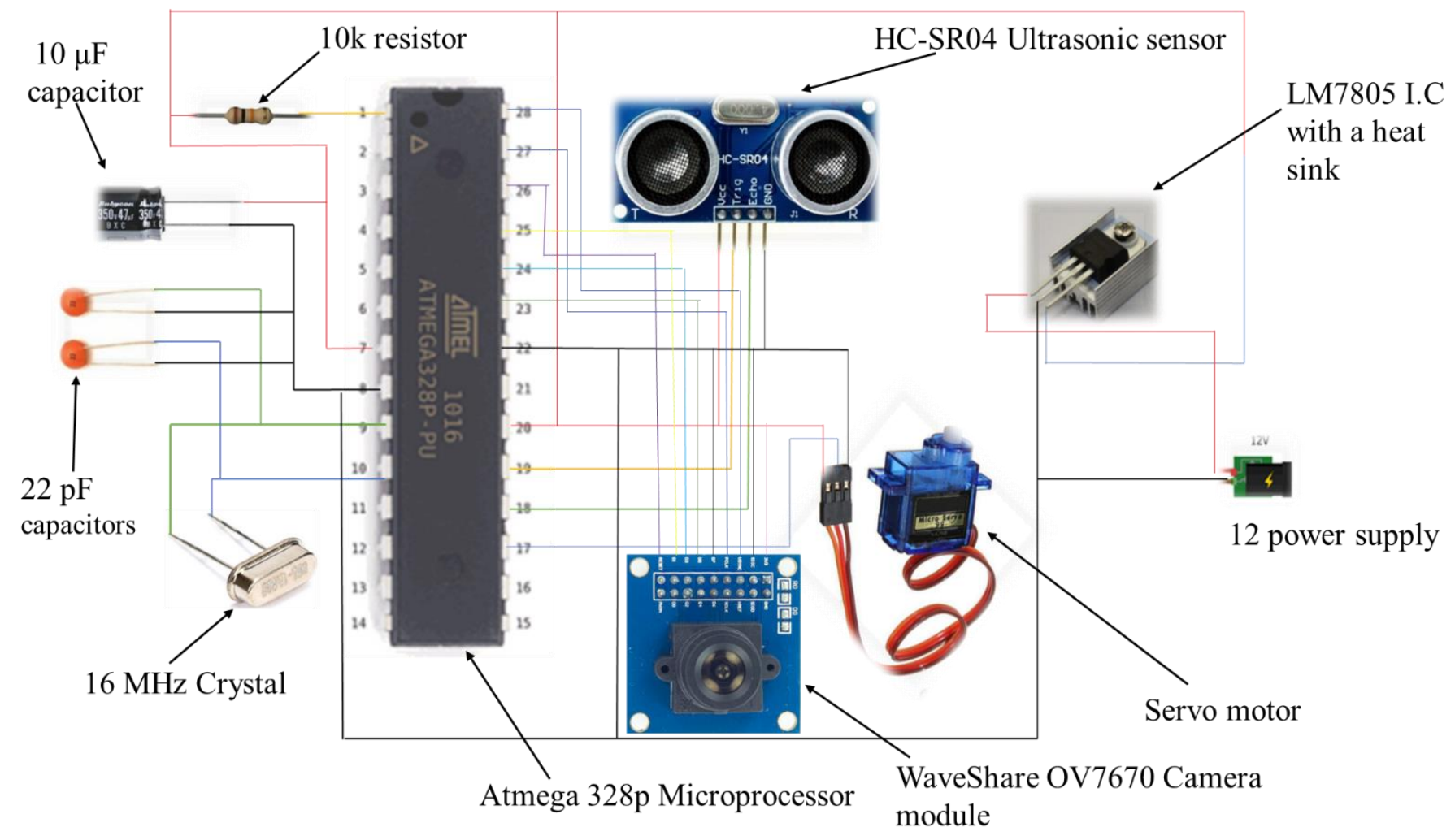

Figure 7: Electrical circuitry of the dispensing process

The monitoring is accomplished by a camera module (Waveshare OV7670 digital camera), which gets triggered parallel to the servomotor and captures a photograph of the individual using the dispenser. It is expected that the images are further passed through a face recognition algorithm and is used to identify and record the hand hygiene events against each individual of an institution. This data may be crucial to check compliance of the hand hygiene protocols followed by each individual in an institution.

The solenoid valve is used for regulating the flow of mixture at times of refilling the sub-tank/tanks. The valve, being an electromechanical device, consumes $12 \mathrm{~V}$ from the battery. A TIP120 transistor is connected in series with the solenoid valve for energizing and de-energizing the valve as per the input signal given by the ultrasonic sensor inside the sub-tank. An ultrasonic sensor perfectly measures the level of a liquid and its presence in dark surrounding won't cause any difference in its 
readings as the sensor working is based on ultrasonic waves (which have nothing to do with light). As soon as the liquid level falls below a threshold value, the Atmega 328p signals the solenoid valve to normally close and after refilling, it would signal the valve to close. Figure 8 presents a schematic of the electrical connections relevant for the refilling circuit.

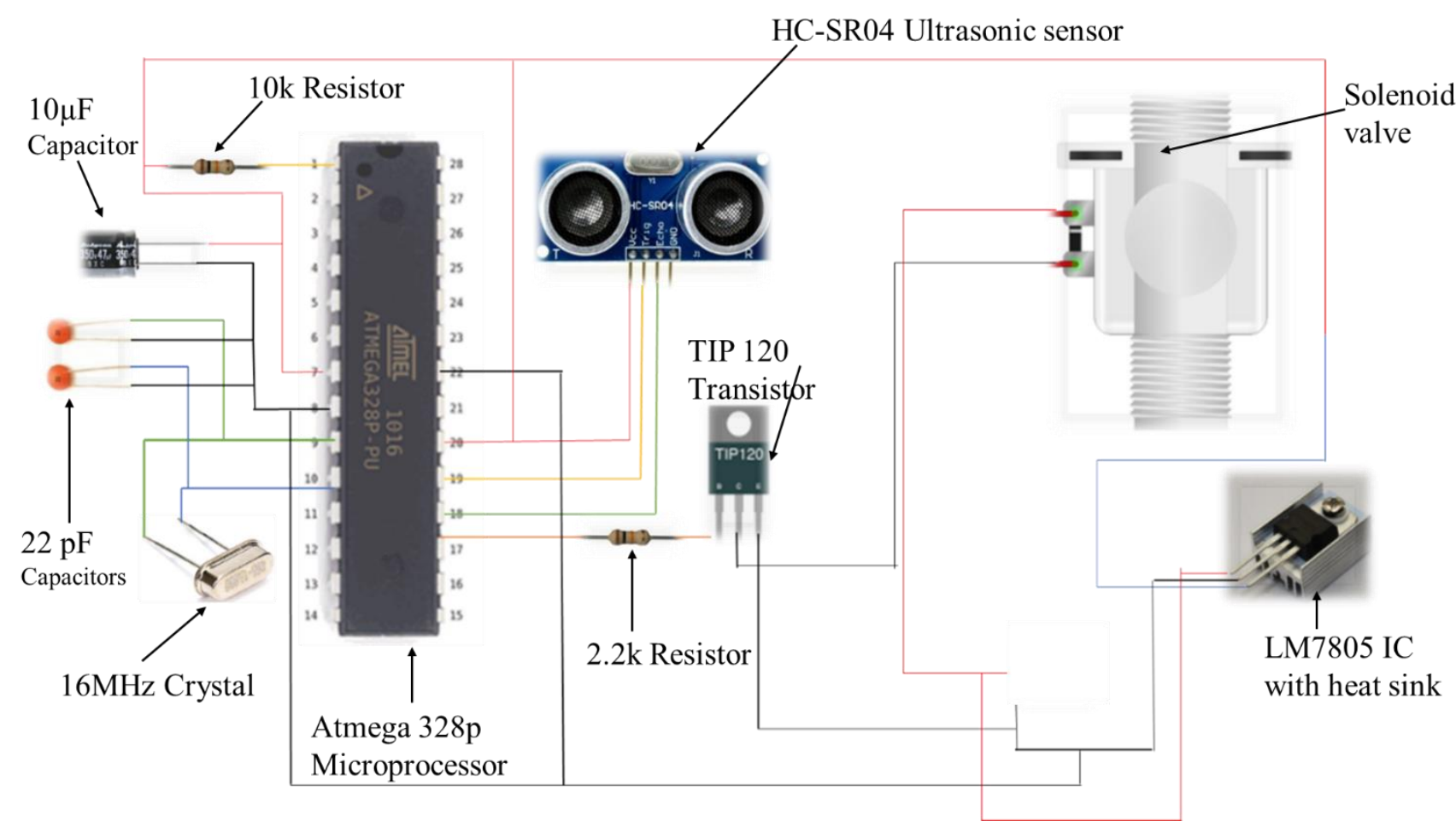

Figure 8: Electrical circuitry of the refilling process

\section{Conclusions}

We propose the design of a fully automated and monitored hand hygiene system, which can be befittingly used in an institutional setting. The proposed design is low-cost, compact and can be installed at the entry points of various important buildings in an educational/healthcare setting. By the integration of a camera module, which triggers in sync with each dispense event, the current design monitors and tracks the hand hygiene regimen followed by each individual in an institutional setting. Further, the proposed product provides for an automated sanitization scheme, in such a way that social distancing norms are not highlighted and thus can effectively help curb the infection spread in community settings.

\section{Funding Sources}

No funding source till the submission of this manuscript.

\section{Conflict of Interest}

The authors declare no conflict of interest in the publication of this manuscript. 


\section{References}

1. Ferguson, N., D. Laydon, G. Nedjati Gilani, N. Imai, K. Ainslie, M. Baguelin, S. Bhatia, A. Boonyasiri, Z. Cucunuba Perez, and G. Cuomo-Dannenburg, Report 9: Impact of nonpharmaceutical interventions (NPIs) to reduce COVID19 mortality and healthcare demand. 2020.

2. Guner, R., I. Hasanoglu, and F. Aktas, COVID-19: Prevention and control measures in community. Turk J Med Sci, 2020. 50(SI-1): p. 571-577.

3. Jefferson, T., R. Foxlee, C. Del Mar, L. Dooley, E. Ferroni, B. Hewak, A. Prabhala, S. Nair, and A. Rivetti, Physical interventions to interrupt or reduce the spread of respiratory viruses: systematic review. Bmj, 2008. 336(7635): p. 77-80.

4. Luby, S.P., M. Agboatwalla, D.R. Feikin, J. Painter, W. Billhimer, A. Altaf, and R.M. Hoekstra, Effect of handwashing on child health: a randomised controlled trial. The Lancet, 2005. 366(9481): p. 225-233.

5. Chen, X., L. Ran, Q. Liu, Q. Hu, X. Du, and X. Tan, Hand Hygiene, Mask-Wearing Behaviors and Its Associated Factors during the COVID-19 Epidemic: A Cross-Sectional Study among Primary School Students in Wuhan, China. Int J Environ Res Public Health, 2020. 17(8).

6. $\quad$ Boyce, J.M., Measuring healthcare worker hand hygiene activity: current practices and emerging technologies. Infect Control Hosp Epidemiol, 2011. 32(10): p. 1016-28.

7. Kampf, G., D. Todt, S. Pfaender, and E. Steinmann, Persistence of coronaviruses on inanimate surfaces and its inactivation with biocidal agents. Journal of Hospital Infection, 2020.

8. Huber, M.A., R.H. Holton, and G.T. Terezhalmy, Cost analysis of hand hygiene using antimicrobial soap and water versus an alcohol-based hand rub. Oral Surgery, Oral Medicine, Oral Pathology, Oral Radiology, and Endodontology, 2005. 99(4): p. 424.

9. Organization, W.H., WHO guidelines on hand hygiene in health care (advanced draft): global safety challenge 2005-2006: clean care is safer care. 2006, World Health Organization.

10. Greenaway, R.E., K. Ormandy, C. Fellows, and T. Hollowood, Impact of hand sanitizer format (gel/foam/liquid) and dose amount on its sensory properties and acceptability for improving hand hygiene compliance. J Hosp Infect, 2018. 100(2): p. 195-201.

11. Vermeil, T., A. Peters, C. Kilpatrick, D. Pires, B. Allegranzi, and D. Pittet, Hand hygiene in hospitals: anatomy of a revolution. J Hosp Infect, 2019. 101(4): p. 383-392.

12. Cheng, V., J. Tai, Y. Ho, and J. Chan, Successful control of norovirus outbreak in an infirmary with the use of alcohol-based hand rub. Journal of Hospital Infection, 2009. 72(4): p. 370-371.

13. Wood, A. and D. Payne, The action of three antiseptics/disinfectants against enveloped and nonenveloped viruses. Journal of Hospital Infection, 1998. 38(4): p. 283-295.

14. Kampf, G., Efficacy of ethanol against viruses in hand disinfection. J Hosp Infect, 2018. 98(4): p. 331-338.

15. Martinello, R.A., J.W. Arbogast, K. Guercia, A.E. Parker, and J.M. Boyce, Nursing preference for alcohol-based hand rub volume. Infect Control Hosp Epidemiol, 2019. 40(11): p. 1248-1252.

16. Pires, D., H. Soule, F. Bellissimo-Rodrigues, A. Gayet-Ageron, and D. Pittet, Hand Hygiene With Alcohol-Based Hand Rub: How Long Is Long Enough? Infect Control Hosp Epidemiol, 2017. 38(5): p. 547-552.

17. WHO, Skin reactions related to hand hygiene, in WHO Guidelines on Hand Hygiene in Health Care: First Global Patient Safety Challenge Clean Care Is Safer Care. 2009: Geneva. p. 14. 